\title{
Weighted Entropy Measure: A New Measure of Information with its Properties in Reliability Theory and Stochastic Orders
}

\author{
M. Mahdy ${ }^{1}$ \\ ${ }^{1}$ Department of Statistics, Mathematics and Insurance, College of Commerce, Benha University, Egypt.
}

\section{ARTICLE INFO}

\section{Article History}

Received April 25, 2017

Accepted May 6, 2018

Keywords

Shannon information

mean residual life

mean reversed life

AMS 2001 Subject Classification

Primary 62N05 Secondary 62E10

\section{ABSTRACT}

The weighted entropy measure is a germane dynamic measure of uncertainty in reliability and survival studies. In this paper, the new results of weighted entropies with some characterizations are provided. Furthermore, we have presented some results for weighted entropy residual and weighted past residual of order statistics with some application of some reliability systems such as a series structure and a parallel structure. In addition, we introduced the lower bound for the weighted residual (past) entropy. Moreover, the stochastic orders based on weighted entropy are presented. Finally, we illustrate the usefulness of the proposed non-parametric estimators of weighted entropy by application to real data.

(C) 2018 The Authors. Published by Atlantis Press SARL. This is an open access article under the CC BY-NC license (http://creativecommons.org/licenses/by-nc/4.0/).

\section{INTRODUCTION}

The weighted distributions have been utilized in many applications such as distributions theory, reliability, probability, ecology, bio-statistics and applied.

Consider the distribution function $G($.) for a random variable $Y \geq 0$ with density function $g($.). Suppose

$l_{Y}=\inf \left\{y \in \mathbb{R}^{1}: G(y)>0\right\}, u_{Y}=\sup \left\{y \in \mathbb{R}^{1}: G(y)<1\right\}, S_{Y}=\left(l_{Y}, u_{Y}\right)$ and $w(.) \in R^{+}$be a weighted function. The weighted random variable $Y^{W}$, having probability density function as:

$$
g^{w}(y)=w(y) g(y) / E[w(y)],-\infty \leq y \leq \infty,
$$

where $E[w(y)] \in \mathbb{R}^{+}$. Let $Y$ represents the life length of a "unit" in reliability studies, and life distribution, with survival function $\bar{G}_{Y}$, hazard rate function $\bar{\varphi}_{G}()=.g_{Y}(.) / \bar{G}_{Y}($.$) , reversed hazard rate \varphi_{G}()=.g_{Y}(.) / G_{Y}($.$) , the geometric vitality function \vartheta(Y)=E(\ln Y \mid Y>0)$ and mean revered residual lifetime as

$$
\theta(t)=\mathbb{E}[\kappa-Y \mid Y \leq \kappa]=\int_{0}^{\kappa} \frac{G_{Y}(u) d u}{G_{Y}(\kappa)}, \kappa \in \mathbb{R}^{+}
$$

As reported by Ebrahimi and Pellery [1] and Asha and Rejeesh [2], the differential entropy $\left(H_{Y}\right)$ demonstrate the expected uncertainty of $g(y)$. In addition, it measures how the distribution spreads over its domain, where there is an inverse relationship between the value of $H_{Y}$ and concentration of the probability mass of $Y$. $H_{Y}$ sometimes called a dynamic measure of uncertainty or Shannon information measure.

The differential entropy of random variable $Y$ can be defined in the continuous case as follows:

$$
H_{Y}=E\left[-\ln g_{Y}(Y)\right]=-\int_{0}^{\infty} g_{Y}(u) \ln g_{Y}(u) d u .
$$

${ }^{*}$ Corresponding author. Email: drmervat.mahdy@fcom.bu.edu.eg 
Khinchin (1957) generalized Eq. (3) as

$$
H_{Y}^{\phi}=E\left[\phi\left(g_{Y}(y)\right)\right]=\int_{0}^{\infty} g_{Y}(u) \phi\left(g_{Y}(u)\right) d u
$$

Di Crescenzo and Longobardi [4] developed the following convex entropy measure:

$$
H^{w}(Y)=-\int_{0}^{\infty} v g_{Y}(v) \ln g_{Y}(v) d v
$$

or equivalently:

$$
H^{w}(Y)=-\int_{0}^{\infty} d y \int_{y}^{\infty} g_{Y}(v) \ln g_{Y}(v) d v
$$

The uncertainty of the residual lifetime is discussed in Di Crescenzo and Longobardi [5], with the following measure:

$$
H(Y, \kappa)=1-E\left[\ln \bar{\varphi}_{G}(Y) \mid Y>\kappa\right]=-\int_{\kappa}^{\infty} \frac{g_{Y}(v)}{\bar{G}(v)} \ln \frac{g_{Y}(v)}{\bar{G}(v)} d v, \kappa \in \mathbb{R}^{+},
$$

where $\kappa \in A=\left\{y \in \mathbb{R}^{+} \mid \bar{G}_{Y}(y)>0\right\}$. In addition, the past entropy has been widely researched. We can measure it as follows:

$$
\bar{H}_{G}(Y, \kappa)=1-E\left[\ln \varphi_{G}(Y) \mid Y<\kappa\right]=-\int_{0}^{\kappa} \frac{g_{Y}(v)}{G(v)} \ln \frac{g_{Y}(v)}{G(v)} d v, \kappa \in \mathbb{R}^{+} .
$$

Di Crescenzo and Longobardi [4] defined the convex residual entropy as

$$
H^{w}(Y, \kappa)=-\int_{\kappa}^{\infty} y \frac{g_{Y}(y)}{\bar{G}(\kappa)} \ln \frac{y g_{Y}(y)}{\bar{G}(\kappa)} d y, \kappa \in \mathbb{R}^{+} .
$$

Furthermore, let $Y_{1}$ and $Y_{2}$ be two random variables with distribution functions $G_{1}$ (.) and $G_{2}($.$) , densities functions g_{Y_{1}}($.$) and g_{Y_{2}}($.$) and$ survival functions $\bar{G}_{1}$ (.) and $\bar{G}_{2}$ (.) respectively. Kullback and Leibler [6] introduced an information distance between two distributions $G_{1}$ and $G_{2}$ as follows:

$$
I_{Y_{1}, Y_{2}}=\int_{0}^{\infty} g_{Y_{1}}(u) \ln \frac{g_{Y_{1}}(u)}{g_{Y_{2}}(u)} d u .
$$

In addition, Ebrahimi and Kirmani [7] have demonstrated that the Kullback-Leibler discrimination information of $Y_{1}$ and $Y_{2}$ at time $\kappa$ can be presented as

$$
I R_{Y_{1}, Y_{2}}(\kappa)=\int_{\kappa}^{\infty} \frac{g_{Y_{1}}(u)}{\bar{G}_{1}(\kappa)} \ln \frac{g_{Y_{1}}(u) / \bar{G}_{1}(\kappa)}{g_{Y}(u) / \bar{G}_{2}(\kappa)} d u
$$

We can use Eq. (5) to distinguish between two residual lifetimes those have both survived up to time $\kappa$, where $I R_{Y_{1}, Y_{2}}(\kappa)$ identifies with the relative entropy of $\left[Y_{1}-\kappa\left|Y_{1}\right\rangle \kappa\right]$ and $\left[Y_{2}-\kappa\left|Y_{2}\right\rangle \kappa\right]$.

The purpose of this study is to develop and add more properties, characterizations, order statistics, some inequalities and stochastic orders of weighted differential entropies measures. In Section 2, definitions, notation, basic properties and characterizations are illustrated. The weighted entropy (residual and past residual) of order statistics with some application of reliability systems such as a series structure and a parallel structure are given in Section 3. In addition, we provided the lower bound for the weighted residual (past) entropy. The stochastic orders based on weighted entropy are developed in Section 4. Lastly, in Section 5, the suggested estimators of weighted entropy are presented. Furthermore, we illustrate the usefulness of the proposed non-parametric estimators of weighted entropy by application to real data.

Throughout this article, the term entropy is used instead of differential entropy and using abbreviation $P L$ for past lifetime, WPE for weighted past residual entropy, WRE for weighted residual entropy, $S S$ for the series system, $P S$ for the parallel system, $S E$ for small than or equal. 


\section{THE WEIGHTED DIFFERENTIAL ENTROPY}

The weighted differential entropy (WDE) defined by Das [8] for random variable $Y$ with weighted function $w(x)=x$ as:

$$
\begin{aligned}
\xi^{w}(Y) & =-\frac{\vartheta^{w}(Y)+\int_{\mathcal{L}} x f_{Y}(x) \ln f_{Y}(x) d x-\mathbb{E}[Y] \ln \mathbb{E}[Y]}{\mathbb{E}[Y]}, \\
& =\frac{H^{w}(Y)}{\mathbb{E}[Y]}+\ln \mathbb{E}[Y]-\frac{\vartheta^{w}(Y)}{\mathbb{E}[Y]},
\end{aligned}
$$

where

$$
\vartheta^{w}(Y)=\int_{\mathcal{L}} x f Y(x) \ln x d x=: \mathbb{E}(Y \ln Y) .
$$

whenever the integral $\int_{\mathcal{L}}\left(u^{\theta} f_{Y}(u) / \mathbb{E}\left(Y^{\theta}\right)\right)\left(1 \vee \mid \ln \left(u^{\theta} f_{Y}(u) / \mathbb{E}\left(Y^{\theta}\right)\right) ।\right) d u<\infty$.

As a general case, we can be defined the generalized the WDE as the following definition:

Definition 2.1. Given a function $y \in \mathcal{L} \mapsto w(y) \geq 0$, and an $R V Y: \mathcal{F} \rightarrow \mathcal{L}$, with a probability density function $g_{Y}($.), survival function $\bar{G}($.) and mean $\mathbb{E}(Y)$. Therefore, the weighted differential entropy with weighted function $w(y)=y^{\theta}$ is defined as

$$
\begin{aligned}
\xi^{w}(Y) & =\mathbb{E}\left[-\ln g_{Y}^{w}(Y)\right]=\int_{\mathcal{L}} g_{Y}^{w}(u) \ln \frac{1}{g_{Y}^{w}(u)} d u, \\
& =-\int_{\mathcal{L}} \frac{u^{\theta} g_{Y}(u)}{\mathbb{E}\left(Y^{\theta}\right)} \ln \frac{u^{\theta} g_{Y}(u)}{\mathbb{E}\left(Y^{\theta}\right)} d u, \\
& =-\frac{1}{\mathbb{E}\left(Y^{\theta}\right)}\left[\theta \int_{\mathcal{L}} u^{\theta} g_{Y}(u) \ln u d u+\int_{\mathcal{L}} u^{\theta} g_{Y}(u) \ln g_{Y}(u) d u-\mathbb{E}\left(Y^{\theta}\right) \ln \mathbb{E}\left(Y^{\theta}\right)\right] .
\end{aligned}
$$

Now, let $Y_{1}, Y_{2}, \ldots, Y_{n}$ be a sample from the distribution $F$ and $n \geq 3$. By using Vasicek [9], express Eq. (2.1) can be rewritten as

$$
\xi^{w}(Y)=\int_{0}^{1} \ln \left\{\frac{\partial}{\partial u} F^{-1}(u)\right\} d u
$$

In addition, Das [8] have defined the weighted residual entropy as

$$
\begin{aligned}
\xi^{w}(Y, \kappa) & =-\int_{\kappa}^{\infty} \frac{g_{Y}^{w}(v)}{\bar{G}^{w}(\kappa)} \ln \frac{g_{Y}^{w}(v)}{\bar{G}^{w}(\kappa)} d v, \\
& =-\frac{1}{E[Y \mid Y>\kappa]} \int_{\kappa}^{\infty} v \frac{g_{Y}(v)}{\bar{G}(\kappa)} \ln \frac{v g_{Y}(v)}{E[Y \mid Y>\kappa] \bar{G}(\kappa)} d v, \kappa \in \mathbb{R}^{+} .
\end{aligned}
$$

If $f_{X}(x)$ is the actual density function of random variable $X$ and $g_{Y}(x)$ is the density function determined by the researcher. Therefore, the weighted inaccuracy measure can be defined as

$$
\mathbb{R}^{w}(X, Y)=-\int_{\mathcal{L}} x f_{X}(x) \ln g_{Y}(x) d x .
$$

Next, we define the relative WDE of two densities.

Definition 2.2. Let $X$ and $Y$ be two random variables with density function, $s \in \mathcal{L} \mapsto f_{X}^{N}(s) \geq 0$ and $s \in \mathcal{L} \mapsto g_{Y}(s) \geq 0$, and mean values $\mathbb{E}_{X}($.$) and \mathbb{E}_{Y}($.$) , respectively. Therefore, the relative weighted differential entropy of g_{Y}(x)$ relative to $f^{N}(x)$ can be defined as

$$
\begin{aligned}
\mathbb{R}\left(X^{w} \| Y\right) & =\mathbb{E}\left[\ln \frac{s f_{X}(s)}{\mathbb{E}_{X}(s) g_{Y}(s)}\right], \\
& =\int_{\mathcal{L}} \frac{v f_{X}(v)}{\mathbb{E}_{X}(X)} \ln \frac{v f_{X}(v)}{\mathbb{E}_{X}(v) g_{Y}(v)} d v .
\end{aligned}
$$

By using Eq. (4), we can define an alternative formulas of $\mathbb{R}\left(X^{w} \| Y\right)$ as follows

$$
\mathbb{R}\left(X^{w} \| Y\right)=\ln \frac{1}{\mathbb{E}_{X}(X)}-\frac{H^{w}(X)}{\mathbb{E}_{X}(X)}-\int_{\mathcal{L}} \frac{x f_{X}(x)}{\mathbb{E}_{X}(x)} \ln x g_{Y}(x) d x .
$$


Note that when $g_{Y}(x) \equiv f_{X}(x)$, then we have

$$
\begin{aligned}
\mathbb{R}\left(X^{w} \| X\right) & =\ln \frac{1}{\mathbb{E}_{X}(X)}-\frac{H^{w}(X)}{\mathbb{E}_{X}(X)}-\int_{\mathcal{L}} \frac{x f_{X}(x)}{\mathbb{E}_{X}(x)} \ln x f_{X}(x) d x, \\
& =\ln \left(\exp \left(-\frac{\vartheta^{w}(X)+2 H^{w}(X)}{\mathbb{E}_{X}(X)}\right) / \mathbb{E}_{X}(X)\right) .
\end{aligned}
$$

Remark 2.1. By using Eqs. (8) and (9) we get the following relation

$$
\mathbb{R}\left(X^{w} \| Y^{w}\right)=\ln \frac{\mathbb{E}_{Y}(X)}{\mathbb{E}_{X}(X)}-\frac{H^{w}(X)}{\mathbb{E}_{X}(X)}+\frac{\mathbb{R}_{X}^{w}(X, Y)}{\mathbb{E}_{X}(X)} .
$$

Furthermore, we can define the divergence between $f^{w}(x)$ and $g_{Y}(x)$ as follows

$$
\mathbb{K}\left(X^{w}, Y\right)=\mathbb{R}\left(X^{w} \| Y\right)+\mathbb{R}^{w}(Y \| X),=\int_{\mathcal{L}}\left(f_{X}^{w}(x)-g_{Y}(x)\right) \ln \frac{f_{X}^{w}(x)}{g_{Y}(x)} d x,
$$

it is a measure of the difficulty of discrimination between them.

Now, let $X$ be RV with beta distribution as follows:

$$
f_{X}(s)=s^{\alpha-1}(1-s)^{\beta-1} / B(\alpha, \beta), s \in \mathcal{L} \in(0,1) .
$$

By using Eq. (3), we get that $H_{X}$ satisfy the following equation:

$$
H_{X}=\ln \left(\frac{B(\alpha, b) \exp ((\alpha+\beta-2) \Psi(\alpha+\beta))}{\exp ((\alpha-1) \Psi(\alpha)+(\beta-1) \Psi(\beta))}\right)
$$

where $B(.$, .) is beta function and $\Psi($.$) is psi function.$

The following theorem states that this relationship actually characterizes the beta distribution.

Characterization Theorem 2.1: Any random variable $Y$ with distribution function $\mathbb{K}$, density function $f_{Y}(x)$, mean $\mathbb{E}[Y]$, mode $\Gamma_{\mathbb{K}}(Y)$, geometric mean $\mathbb{G}[Y]$, entropy function $H_{Y}$ and weighted differential entropy $\xi^{w}(Y)$ satisfying the following relationship:

$$
\xi^{w}(Y)=H_{Y}-(\alpha-1)\left[\frac{\Gamma_{\mathbb{K}}(Y)+\mathbb{E}[Y]}{\Gamma_{\mathbb{K}}(Y)}\right]+\ln \mathbb{E}[Y]-\ln (\mathbb{G}[Y])+((\mathbb{E}[Y]-1) / \alpha),
$$

is either degenerate or $Y$ has a beta distribution. Indeed, the degenerate case should be subsumed in the beta distribution with $(\alpha, \beta) \in R^{+}$.

Proof. By using the following integral formula which is taken from Gradshteyn and Ryzhik ([10], formula 4.253(1), pp. 538):

$$
\int_{\mathcal{L}} y^{\theta-1}\left(1-y^{c}\right)^{\lambda-1} \ln y d y=\frac{1}{c^{2}} B\left(\frac{\theta}{c}, \lambda\right)\left(\Psi\left(\frac{\theta}{c}\right)-\Psi\left(\frac{\theta}{c}+\lambda\right)\right),
$$

provided that $\operatorname{Re}(\theta)>0, \operatorname{Re}(\lambda)>0, c>0$. We denote the beta function with the symbol $B(\cdot, \cdot)$ and the digamma function with $\Psi(\cdot)$. Therefore, we have

$$
\begin{aligned}
\vartheta^{w}(Y) & =\int_{\mathcal{L}} x f_{X}(x) \ln x d x, \\
& =\frac{1}{B(\alpha, \beta)}[B(\alpha+1, \beta)(\Psi(\alpha+1)-\Psi(\alpha+\beta+1))], \\
& =\mathbb{E}[Y](\Psi(\alpha+1)-\Psi(\alpha+\beta+1)) .
\end{aligned}
$$

By using Example 2.3 in Di Crescenzo and Longobard ([4], pp.682), Eq. (4) and the recurrence relation of the digamma function, we can rewrite $H^{w}(Y)$ as follows:

$$
H^{w}(Y)=\mathbb{E}[Y]\left[\begin{array}{c}
\ln B(\alpha, \beta)+(1-\alpha)\left(\Psi(\alpha)+\frac{1}{\alpha}\right) \\
+(\alpha+\beta-2)\left(\Psi(\alpha+\beta)+\frac{1}{\alpha+\beta}\right)+\Psi(\beta)(1-\beta)
\end{array}\right]
$$

We can reduce Eq. (11) as,

$$
H^{w}(Y)=\mathbb{E}[Y]\left[H_{Y}+(1-\alpha) \frac{1}{\alpha}+\frac{(\alpha+\beta-2)}{(\alpha+\beta)}\right],=\mathbb{E}[Y]\left[H_{Y}-(\alpha-1)\left[\frac{\Gamma_{\mathbb{K}}(Y)+\mathbb{E}[Y]}{\Gamma_{\mathbb{K}}(Y)}\right]\right] .
$$


This is true for $f\left(\Gamma_{\mathbb{K}}().\right)=\max _{-\infty<x<\infty} f_{X}($.$) . Furthermore by Eqs. (6) and (10) we have$

$$
\begin{aligned}
\xi^{w}(Y) & =H_{Y}-(\alpha-1)\left[\frac{\Gamma_{\mathbb{K}}(Y)+\mathbb{E}[Y]}{\Gamma_{\mathbb{K}}(Y)}\right]+\ln \mathbb{E}[Y]-(\Psi(\alpha+1)-\Psi(\alpha+\beta+1)), \\
& =H_{Y}-(\alpha-1)\left[\frac{\Gamma_{\mathbb{K}}(Y)+\mathbb{E}[Y]}{\Gamma_{\mathbb{K}}(Y)}\right]+\ln \mathbb{E}[Y]-\ln (\mathbb{G}[Y])+\frac{1}{\alpha}(\mathbb{E}[Y]-1) .
\end{aligned}
$$

In next results we study the closure transformation property of the weighted entropy. We can now proceed analogously to Di Crescenzo and Longobardi [4] and introduce the following theorem.

Theorem 2.2. Suppose $U$ is $R V$ with density function $f_{U}($.$) and \psi(U)$ is strongly convex, strictly increasing, continuous and differentiable function with derivative $\frac{d}{d u} \psi(u)$. Then

$$
\xi^{w}(\psi(U))=\xi_{1}^{w}\left(U \mid \psi^{-1}(0) \leq U \leq \psi^{-1}(\infty)\right)+\mathbb{E}^{w}\left[\ln \left|\frac{d}{d x} \psi(x)\right| \psi^{-1}(0) \leq U \leq \psi^{-1}(\infty) \mid\right]
$$

where $E^{w}(U)=\int_{0}^{\infty} v f_{U}^{w}(v) d v$

Proof. From Eq. (6) we have

$$
\xi^{w}(\psi(U))=-\int_{0}^{\infty} f_{U}^{w}\left(\psi^{-1}(u)\right)\left|\frac{d}{d u} \psi^{-1}(u)\right| \ln f_{U}^{w}\left(\psi^{-1}(u)\right)\left|\frac{d}{d u} \psi^{-1}(u)\right| d u .
$$

We will make the following assumptions:

1. $\psi(u)$ is monotonically increasing in $u$.

2. $v=\psi(u)$,

Therefore, it clear that

$$
\begin{aligned}
\xi^{w}(\psi(U)) & =-\int_{\psi^{-1}(0)}^{\psi^{-1}(\infty)} f_{U}^{w}(v) \ln \left(f_{U}^{w}(v)\left|\frac{d}{d v} \psi(v)\right|^{-1}\right) d v \\
& =\int_{\psi^{-1}(0)}^{\psi^{-1}(\infty)} f_{U}^{w}(v) \ln \left|\frac{d}{d v} \psi(v)\right| d v+\xi^{w}\left(U \mid \psi^{-1}(0) \leq U \leq \psi^{-1}(\infty)\right) .
\end{aligned}
$$

Hence the proof is completed.

Proposition 2.3. Let $\phi(U)=\alpha U^{\beta}$ whereas $\alpha, \beta>0$. From this we deduce that

$$
\xi^{w}(\phi(U))=\ln \alpha \beta+\frac{(\beta-1) \vartheta^{w}(U)}{\mathbb{E}(U)}+\xi^{w}(U) .
$$

Proof. From Eq. (6) we have

$$
\begin{aligned}
\xi^{w}(\phi(U)) & =\int_{0}^{\infty} f^{w}(v) \ln \left|\alpha \beta v^{\beta-1}\right| d v-\int_{0}^{\infty} f^{w}(v) \ln f^{w}(v) d v \\
& =\ln \alpha \beta+(\beta-1) \int_{0}^{\infty} f^{w}(v) \ln v d v+\xi^{w}(U)
\end{aligned}
$$

By using Eq. (7), we get the required results.

From Definitions (2.1), it is easy to obtain the following characterizations:

Example 2.1: Suppose $U$ be a random variable having Log-Normal with the following density function

$$
f_{U}(u)=\frac{1}{\sqrt{2 \pi} \sigma u} \exp \left(-(\ln u-\ln \mu)^{2} / 2 \sigma^{2}\right), \mu, \sigma, u>0,
$$

with parameter $\mu, \sigma>0$. From Eq. (7) we get,

$$
\vartheta^{w}(U)=\int_{0}^{\infty} v \frac{1}{\sqrt{2 \pi} \sigma v} \exp \left(-(\ln v-\ln \mu)^{2} / 2 \sigma^{2}\right) \ln v d v .
$$


Set $u=\ln v-\ln \mu$, then we have

$$
\vartheta^{w}(U)=\frac{2}{\sqrt{2 \pi} \sigma} \exp (2 \ln \mu) \int_{0}^{\infty} \exp \left(2 u-\frac{u^{2}}{2 \sigma^{2}}\right) d u
$$

It is follows from Gradshteyn and Ryzhik ([10], formula 3.322(1)) that

$$
\int_{a}^{\infty} \exp \left(-\frac{x^{2}}{4 \mu}-b x\right) d x=\sqrt{\pi \mu} \exp \left(\mu b^{2}\right)\left(1-\Phi\left(b \sqrt{\mu}+\frac{a}{2 \sqrt{\mu}}\right)\right),[\operatorname{Re} \mu>0, a \geq 0] .
$$

Therefore,

$$
\vartheta^{w}(U)=\exp \left(2\left(\ln \mu+\sigma^{2}\right)\right)(1-\Phi(-2 \sqrt{1 / 2} \sigma)) .
$$

In addition,

$$
\begin{aligned}
H^{w}(U) & =-\int_{0}^{\infty} v \frac{1}{\sqrt{2 \pi \sigma v}} \exp \left(-(\ln v-\ln \mu)^{2} / 2 \sigma^{2}\right)\left[-\ln \sqrt{2 \pi \sigma}-\ln v-\frac{(\ln v-\ln \mu)^{2}}{2 \sigma^{2}}\right] d v, \\
& =\ln \sqrt{2 \pi} \sigma \mathbb{E}[U]+\vartheta^{w}(U)+\int_{0}^{\infty} v \frac{1}{\sqrt{2 \pi} \sigma v} \exp \left(-(\ln v-\ln \mu)^{2} / 2 \sigma^{2}\right) \frac{(\ln v-\ln \mu)^{2}}{2 \sigma^{2}} d v,
\end{aligned}
$$

with the same way, set $u=\ln v-\ln \mu$ and by using formula 3.462(1) in Gradshteyn and Ryzhik [10] we have

$$
\begin{gathered}
\int_{0}^{\infty} v \frac{1}{\sqrt{2 \pi} \sigma v} \exp \left(-(\ln v-\ln \mu)^{2} / 2 \sigma^{2}\right) \frac{(\ln v-\ln \mu)^{2}}{2 \sigma^{2}} d v \\
=\left(\frac{1}{\sigma^{2}}\right)^{(-3 / 2)+3} \frac{2 \mu}{\sqrt{2 \pi}} \exp \left(\frac{\sigma^{2}}{4}\right) D_{-3}(-\sigma)
\end{gathered}
$$

Therefore,

$$
\begin{aligned}
H^{w}(X)= & \ln (\sqrt{2 \pi} \sigma) \mu \exp \left(\sigma^{2} / 2\right)+\exp \left(2\left(\ln \mu+\sigma^{2}\right)\right)(1-\Phi(-2 \sqrt{1 / 2} \sigma)) \\
& +\left(\frac{1}{\sigma^{2}}\right)^{(-3 / 2)+3} \frac{2 \mu}{\sqrt{2 \pi}} \exp \left(\frac{\sigma^{2}}{4}\right) D_{-3}(-\sigma), \\
= & \ln (\sqrt{2 \pi} \sigma) \mathbb{E}[X]+\vartheta^{w}(X)+\sigma^{-3} \frac{2 \mu}{\sqrt{2 \pi}} \exp \left(\frac{\sigma^{2}}{4}\right) D_{-3}(-\sigma),
\end{aligned}
$$

where $\Phi($.$) is Error function, \varpi(\mu, \sigma)=\left(\mu \exp \left(\sigma^{2} / 2\right)\right)^{-1}$ and $D_{x}(y)=2^{x / 2} \exp \left(-y^{2} / 4\right) F\left(\frac{-x}{2}, \frac{1}{2}, \frac{y^{2}}{2}\right)$ is Parabolic cylinder function. We denote a confluent hypergeometric function of the first kind with the symbol $F(., .,$.$) . Further,$

$$
\xi^{w}(U)=\varpi(\mu, \sigma) \alpha(\mu, \sigma)-\ln \varpi(\mu, \sigma)-\varpi(\mu, \sigma) \exp \left(2\left(\ln \mu+\sigma^{2}\right)\right)(1-\Phi(-2 \sqrt{1 / 2} \sigma)),
$$

where $\alpha(\mu, \sigma)=\ln \sqrt{2 \pi} \sigma \mu \exp \left(\sigma^{2} / 2\right)+\exp \left(2\left(\ln \mu+\sigma^{2}\right)\right)(1-\Phi(-2 \sqrt{1 / 2} \sigma))+\left(\frac{1}{\sigma^{2}}\right)^{(-3 / 2)+3} \frac{2 \mu}{\sqrt{2 \pi}} \exp \left(\frac{\sigma^{2}}{4}\right) D_{-3}(-\sigma)$.

Furthermore,

$$
\mathbb{R}\left(X^{w} \| X\right)=\ln \left(\exp \left(-\beta_{1}(\mu, \sigma)+2 \beta_{2}(\mu, \sigma) \varpi(\mu, \sigma)\right) / \varpi(\mu, \sigma)\right)
$$

where:

1. $\beta_{1}(\mu, \sigma)=\exp \left(2\left(\ln \mu+\sigma^{2}\right)\right)(1-\Phi(-2 \sqrt{1 / 2} \sigma))$;

2. $\beta_{2}(\mu, \sigma)=\ln (\sqrt{2 \pi} \sigma) \mathbb{E}[X]+\vartheta^{w}(X)+\left(\frac{1}{\sigma^{2}}\right)^{(-3 / 2)+3} \frac{2 \mu}{\sqrt{2 \pi}} \exp \left(\frac{\sigma^{2}}{4}\right) D_{-3}(-\sigma)$ 
Example 2.2: Let $X$ be random variable having Chi distribution with density function

$f_{X}(x)=\frac{2(\pi / 2)^{\pi / 2}}{\gamma^{\pi} \Gamma(\pi / 2)} x^{\pi-1} \exp \left(-x^{2}\left(\pi /\left(2 \gamma^{2}\right)\right)\right), \gamma, x>0, \pi$ is a positive integer, with parameter $\mu, \gamma>0$. From Eq. (7) we have

$$
\vartheta^{w}(X)=\frac{2(\pi / 2)^{\pi / 2}}{\gamma^{\pi} \Gamma(\pi / 2)} \int_{0}^{\infty} x^{\pi} \exp \left(-x^{2}\left(\pi /\left(2 \gamma^{2}\right)\right)\right) \ln x d x
$$

take $u=x^{2} \pi /\left(2 \gamma^{2}\right)$, then we get

$$
\vartheta^{w}(X)=\frac{\gamma(1 / 2 \pi)^{1 / 2}}{\Gamma(\pi / 2)} \int_{0}^{\infty} u^{\frac{\pi}{2}-0.5} \exp (-u) \ln \left(\left(u /\left(\pi /\left(2 \gamma^{2}\right)\right)\right)^{1 / 2}\right) d u
$$

By using Gradshteyn and Ryzhik ([10], 4.352(1)) for evaluate the below formula, we have the following result:

$$
\vartheta^{w}(X)=\frac{\gamma(1 / 2 \pi)^{1 / 2}}{\Gamma(\pi / 2)} \Gamma\left(\frac{\pi+1}{2}\right)\left[\left(\psi\left(\frac{\pi+1}{2}\right)\right)-\ln \left(\pi /\left(2 \gamma^{2}\right)\right)\right] .
$$

In addition, by Eq. (4) we have

$$
H^{w}(X)=-\int_{0}^{\infty} x \frac{2(\pi / 2)^{\pi / 2}}{\gamma^{\pi} \Gamma(\pi / 2)} x^{\pi-1} \exp \left(-x^{2}\left(\pi /\left(2 \gamma^{2}\right)\right)\right)\left[\ln \frac{2(\pi / 2)^{\pi / 2}}{\gamma^{\pi} \Gamma(\pi / 2)}+(\pi-1) \ln x-\frac{x^{2} \pi}{2 \gamma^{2}}\right] d x
$$

since $\mathbb{E}(X)=\gamma \sqrt{\frac{2}{\pi}} \frac{\Gamma\left(\frac{\pi+1}{2}\right)}{\Gamma\left(\frac{\pi}{2}\right)}$. With this substitution we obtain

$$
H^{w}(X)=\begin{aligned}
& \pi_{2}-\pi_{1}(\pi-1) \int_{0}^{\infty} v^{\pi} \exp \left(-v^{2}\left(\pi /\left(2 \gamma^{2}\right)\right)\right) \ln v d v \\
& +\pi_{3} \int_{0}^{\infty} v^{\pi+2} \exp \left(-v^{2}\left(\pi /\left(2 \gamma^{2}\right)\right)\right) d v
\end{aligned}
$$

where $\pi_{1}=2(\pi / 2)^{\pi / 2} /\left(\gamma^{\pi} \Gamma(\pi / 2)\right), \pi_{2}=-\ln \left(\pi_{1}\right) \gamma\left(\Gamma\left(\frac{\pi+1}{2}\right) / \Gamma\left(\frac{\pi}{2}\right)\right) \sqrt{2 / \pi}, \pi_{3}=\pi /\left(2 \gamma^{2}\right) \pi_{1}$. Direct calculations give $H^{w}(X)=\frac{\gamma(\pi+1) \Gamma\left(\frac{\pi+1}{2}\right)}{2(\pi / 2)^{1 / 2} \Gamma(\pi / 2)}-\ln \left(\frac{2(\pi / 2)^{\pi / 2}}{\gamma^{\pi} \Gamma(\pi / 2)}\right) \frac{\gamma \sqrt{\frac{2}{\pi}} \Gamma\left(\frac{\pi+1}{2}\right)}{\Gamma\left(\frac{\pi}{2}\right)}-\frac{2(\pi / 2)^{\pi / 2}(\pi-1)}{\gamma^{\pi} \Gamma(\pi / 2)} \int_{0}^{\infty} x^{\pi} \exp \left(-x^{2}\left(\pi /\left(2 \gamma^{2}\right)\right)\right) \ln x d x$

Again, we the same way and continuing the simplification, we can conclude that

$$
H^{w}(X)=-\left(\gamma \Gamma\left(\frac{\pi+1}{2}\right) / \Gamma(\pi / 2)\right)\left(\begin{array}{l}
\ln \left(\frac{2(\pi / 2)^{\pi / 2}}{\gamma^{\pi} \Gamma(\pi / 2)}\right) \sqrt{\frac{2}{\pi}} \\
+\frac{2(\pi-1)}{4 \sqrt{(\pi / 2)}}\left[\psi\left(\frac{\pi+1}{2}\right)-\ln \left(\pi /\left(2 \gamma^{2}\right)\right)\right]-\frac{(\pi+1)}{2(\pi / 2)^{1 / 2}}
\end{array}\right) .
$$

Therefore,

$$
\begin{gathered}
\xi^{w}(X)=-\ln \left(2 / \gamma^{\pi}\right)-\frac{2(\pi-1)}{4}\left[\psi\left(\frac{\pi+1}{2}\right)-\ln \left(\pi /\left(2 \gamma^{2}\right)\right)\right]+\frac{(\pi+1)}{2}(1-\ln (\pi / 2)) \\
+\ln \left(\gamma \Gamma\left(\frac{\pi+1}{2}\right)\right)-\psi\left(\frac{\pi+1}{2}\right)+\ln \pi /\left(2 \gamma^{2}\right)
\end{gathered}
$$

Continuing the simplification

$$
\begin{gathered}
\xi^{w}(X)=\ln \left(\frac{\pi \gamma^{\pi-1}}{4}\right)-\frac{2(\pi-1)}{4}\left[\psi\left(\frac{\pi+1}{2}\right)-\ln \left(\pi /\left(2 \gamma^{2}\right)\right)\right] \\
+\frac{(\pi+1)}{2}(1-\ln (\pi / 2))+\ln \Gamma\left(\frac{\pi+1}{2}\right)-\psi\left(\frac{\pi+1}{2}\right) .
\end{gathered}
$$


Moreover,

$$
\mathbb{R}\left(X^{w} \| X\right)=-\frac{\pi \alpha_{1}\left[\left(\psi\left(\frac{\pi+1}{2}\right)\right)-\ln \left(\pi /\left(2 \gamma^{2}\right)\right)\right]+2 H^{w}(X)}{\alpha_{1}}-\ln \left(\alpha_{1}\right),
$$

where $\alpha_{1}=\gamma \Gamma\left(\frac{\pi+1}{2}\right) / \Gamma\left(\frac{\pi}{2}\right) \sqrt{2 / \pi}$. Continuing the simplification

$$
\mathbb{R}^{w}\left(X^{w} \| X\right)=\left(\frac{4 \pi+1}{2}\right) \ln \left(\pi /\left(2 \gamma^{2}\right)\right)+2 \ln 2+\psi\left(\frac{\pi+1}{2}\right)(\pi-2)-(\pi+1)-\ln \left(\Gamma\left(\frac{\pi+1}{2}\right) \Gamma\left(\frac{\pi}{2}\right)\right) .
$$

Example 2.3: A random variable $U$ has a Laplace $(\alpha)$, if it has density function as follows

$$
f_{U}(u)=\frac{1}{2} \alpha \exp (-\alpha|u|), \alpha, \infty>u>-\infty,
$$

with parameter $\alpha>0$. From Eq. (7) we obtain,

$$
\vartheta^{w}(U)=\int_{-\infty}^{\infty} v \frac{1}{2} \alpha \exp (-\alpha|v|) \ln v d v=(\psi(2)-\ln \alpha) / \alpha,
$$

Furthermore, direct calculations give

$$
\begin{aligned}
H^{w}(U) & =-\int_{0}^{\infty} x \alpha \exp (-\alpha x)\left(\ln \frac{\alpha}{2}-\alpha x\right) d x, \\
& =\frac{2-\ln \alpha+\ln 2}{\alpha} \text { nats }=\frac{1+H_{U}}{\alpha} \text { nats. }
\end{aligned}
$$

Since $\mathbb{E}[U]=0$, we get that $\xi_{1}^{w}(U)$ and $\mathbb{R}_{1}^{w}\left(U^{w} \| U\right)$ can not be found.

\section{CONNECTION TO RELIABILITY THEORY}

Suppose $U_{1}, U_{2}, \ldots, U_{n}$ be i.i.d. lifetimes with probability density function $g($.$) , distribution \mathbb{K}($.$) , survival function \overline{\mathbb{K}}($.$) and reversed hazard$ rate $\varphi_{\mathbb{K}}$ (.). Therefore, the probability that any two (or more) observation in random sample take the same magnitude (the same value is equal to zero). Therefore, there exists a unique ordered arrangement of the sample observation according to magnitude. Let $0 \leq U_{(1)} \leq$ $U_{(2)} \leq \ldots \leq U_{(n)}<\infty$ be the corresponding order statistics. Therefore, $U_{(r)}$ defines the lifetime of an $(n-r+1)$ out of $n$ system. Write $g_{(r)}($.$) ,$ $\mathbb{K}_{(r)}(),. \varphi_{(r)}($.$) and \bar{\varphi}_{(r)}($.$) as the distribution function, the probability density function, the RHR function of U_{(r)}$ and the hazard rate of $U_{(r)}$ respectively. Then we have

$$
\begin{aligned}
& g_{(r)}(\kappa)=C_{r}[\mathbb{K}(\kappa)]^{r-1}[\overline{\mathbb{K}}(\kappa)]^{n-r} g(\kappa), \kappa \in \mathbb{R}^{+}, \\
& \mathbb{K}_{(r)}(\kappa)=\sum_{i=r}^{n}\left(\begin{array}{c}
n \\
i
\end{array}\right)[\mathbb{K}(\kappa)]^{i}[\overline{\mathbb{K}}(\kappa)]^{n-i}, \\
& \varphi_{(r)}(\kappa)=C_{r} \varphi_{\mathbb{K}}(\kappa) \beta^{r} /\left(\sum_{i=r}^{n}\left(\begin{array}{c}
n \\
i
\end{array}\right) \beta^{i}\right),
\end{aligned}
$$

and

$$
\bar{\varphi}_{(r)}(\kappa)=g_{(r)}(\kappa) / \overline{\mathbb{K}}_{(r)}(\kappa)
$$

where $C_{r}=\frac{n !}{(r-1) !(n-r) !}$ and $\beta^{x}=(\mathbb{K}(\kappa) / \bar{K}(\kappa))^{x}$. The weighted residual entropy of order statistics $U_{r}$ is given by

$$
\begin{aligned}
\xi_{1}^{w}\left(U_{(r)}, \kappa\right)=-\int_{\kappa}^{\infty} \frac{g_{(r)}^{w}(u)}{\overline{\mathbb{K}}_{(r)}^{w}(\kappa)} \ln \frac{g_{(r)}^{w}(u)}{\overline{\mathbb{K}}_{(r)}^{w}(\kappa)} d u,=\frac{-1}{\mathbb{E}\left[U_{(r)} \mid U_{(r)}>\kappa\right]} \int_{\kappa}^{\infty} \frac{y g_{(r)}(y)}{\overline{\mathbb{K}}_{(r)}(\kappa)} \\
\quad \times \ln \frac{y g_{(r)}(y)}{\mathbb{E}\left[U_{(r)} \mid U_{(r)}>\kappa\right] \overline{\mathbb{K}}_{(r)}(\kappa)} d y .
\end{aligned}
$$


Alternatively,

$$
\begin{aligned}
\xi_{1}^{w}\left(U_{(r)}, \kappa\right)= & \frac{-1}{\mathbb{E}\left[U_{(r)} \mid U_{(r)}>\kappa\right]} \\
& \times \int_{\kappa}^{\infty} \frac{y g_{(r)}(y)}{\overline{\mathbb{K}}_{(r)}(\kappa)} \ln \frac{y \bar{\varphi}_{(r)}(y) \overline{\mathbb{K}}_{(r)}(y)}{\mathbb{E}\left[U_{(r)} \mid U_{(r)}>\kappa\right] \overline{\mathbb{K}}_{(r)}(\kappa)} d y, \\
= & \ln \left[\mathbb{E}\left[U_{(r)} \mid U_{(r)}>\kappa\right] \overline{\mathbb{K}}_{(r)}(\kappa)\right] \\
& -\frac{1}{\mathbb{E}\left[U_{(r)} \mid U_{(r)}>\kappa\right]} \int_{\kappa}^{\infty} \frac{y g_{(r)}(y)}{\overline{\mathbb{K}}_{(r)}(\kappa)} \ln \left(y \bar{\varphi}_{(r)}(y) \overline{\mathbb{K}}_{(r)}(y)\right) d y .
\end{aligned}
$$

Now, we can proceed analogously to treatment of the weighted entropy of the order statistics of PL as follows

$$
\begin{aligned}
\xi_{2}^{w}\left(U_{(r)}, \kappa\right) & =-\int_{0}^{\kappa} \frac{g_{(r)}^{w}(v)}{\mathbb{K}_{(r)}^{w}(\kappa)} \ln \frac{g_{(r)}^{w}(v)}{\mathbb{K}_{(r)}^{w}(\kappa)} d v, \\
& =\frac{-1}{\mathbb{E}\left[U_{(r)} \mid U_{(r)}<\kappa\right]} \int_{0}^{\kappa} \frac{v g_{(r)}(v)}{\mathbb{K}_{(r)}(\kappa)} \ln \frac{v g_{(r)}(v)}{\mathbb{E}\left[U_{(r)} \mid U_{(r)}<\kappa\right] \mathbb{K}_{(r)}^{w}(\kappa)} d v,
\end{aligned}
$$

which is equivalent to

$$
\xi_{2}^{w}\left(U_{(r)}, \kappa\right)=\frac{-1}{\mathbb{E}\left[U_{(r)} \mid U_{(r)}<\kappa\right]} \int_{0}^{\kappa} \frac{x g_{(r)}(x)}{\mathbb{K}_{(r)}(\kappa)} \ln \frac{x \varphi_{(r)}(x) \overline{\mathbb{K}}_{(r)}(x)}{\mathbb{E}\left[U_{(r)} \mid U_{(r)}<\kappa\right] \mathbb{K}_{(r)}^{w}(\kappa)} d x
$$

Direct calculations give

$$
\begin{gathered}
\xi_{2}^{w}\left(U_{(r)}, \kappa\right)=\ln \left[\mathbb{E}\left[U_{(r)} \mid U_{(r)}<\kappa\right] \mathbb{K}_{(r)}(\kappa)\right] \\
-\frac{1}{\mathbb{E}\left[U_{(r)} \mid U_{(r)}<\kappa\right]} \int_{0}^{\kappa} \frac{v g_{(r)}(v)}{\mathbb{K}_{(r)}^{w}(\kappa)(\kappa)} \ln \left(v \varphi_{(r)}(v) \mathbb{K}_{(r)}(v)\right) d v,
\end{gathered}
$$

for all $\kappa \geq 0$.

\subsection{A Series Structure}

It is to be noted that $U_{(1)}$ represents an age of the series system. By using Eq. (13), with simple calculation, we have the weighted the residual entropy of $U_{(1)}$ as

$$
\begin{aligned}
\xi_{1}^{w}\left(U_{(1)}, \kappa\right)= & \ln \left[\mathbb{E}\left[U_{(1)} \mid U_{(1)}>\kappa\right] \overline{\mathbb{K}}_{(1)}(\kappa)\right] \\
& -\frac{1}{\mathbb{E}\left[U_{(1)} \mid U_{(1)}>\kappa\right]} \int_{\kappa}^{\infty} \frac{v g_{(1)}(v)}{\overline{\mathbb{K}}_{(1)}(\kappa)} \ln \left(v \bar{\varphi}_{(1)}(v) \overline{\mathbb{K}}_{(1)}(v)\right) d v, \\
= & \ln \left[\mathbb{E}\left[U_{(1)} \mid U_{(1)}>\kappa\right] \overline{\mathbb{K}}_{(1)}(\kappa)\right] \\
& -\frac{1}{\mathbb{E}\left[U_{(1)} \mid U_{(1)}<\kappa\right]} \int_{0}^{\infty} \frac{v n[\overline{\mathbb{K}}(v)]^{n-1} g(v)}{[\overline{\mathbb{K}}(\kappa)]^{n} \ln \left(v \bar{\varphi}_{(1)}(v)[\overline{\mathbb{K}}(v)]^{n}\right) d v .}
\end{aligned}
$$

It follows from Proposition 1 in Bairamov et al. [11], and definition of mean residual lifetime of $(n-k+1)$-out-of- $n$ system in Asadi and Bayramoglu [12] that

$$
\mathbb{E}\left[U_{(1)} \mid U_{(1)}>\kappa\right]=\frac{\int_{\kappa}^{\infty} \overline{\mathbb{K}}_{1}(v) d v}{\overline{\mathbb{K}}_{1}(\kappa)}=\mathbb{M}_{1}(\kappa) s a y,
$$


and

$$
\overline{\mathbb{K}}(\kappa)=\left(\frac{\mathbb{M}_{1}(0)}{\mathbb{M}_{1}(\kappa)} \exp \left(-\int_{0}^{\kappa} \mathbb{M}_{1}^{-1}(\kappa)\right)\right)^{1 / n},
$$

Then, Eq. (15) can be written in the following form

$$
\xi_{1}^{w}\left(U_{(1)}, \kappa\right)=\ln \left(\mathbb{M}_{1}(0) \exp \left(-\int_{0}^{\kappa} \mathbb{M}_{1}^{-1}(\kappa)\right)\right)+\frac{\int_{\kappa}^{\infty} \frac{v n[\overline{\mathbb{K}}(v)]^{n-1} g(v)}{[\overline{\mathbb{K}}(\kappa)]^{n}} \ln \left(v \bar{\varphi}_{(1)}(v)[\overline{\mathbb{K}}(v)]^{n}\right) d v}{\mathbb{M}_{1}(0) \exp \left(-\int_{0}^{\kappa} \mathbb{M}_{1}^{-1}(\kappa)\right)} .
$$

Similarly, by Eq. (14), the WPE of $U_{(1)}$ follows

Eq. (12) implies that

$$
\begin{aligned}
\xi_{2}^{w}\left(U_{(1)}, \kappa\right)= & \ln \left[\mathbb{E}\left[U_{(1)} \mid U_{(1)}<\kappa\right] \mathbb{K}_{(1)}(\kappa)\right] \\
& -\frac{1}{\mathbb{E}\left[U_{(1)} \mid U_{(1)}<\kappa\right]} \int_{0}^{\kappa} \frac{v g_{(1)}(v)}{\mathbb{K}_{(1)}(\kappa)} \ln \left(v \varphi_{(1)}(v) \mathbb{K}_{(1)}(v)\right) d v, \\
= & \ln \left[\mathbb{E}\left[U_{(1)} \mid U_{(1)}<\kappa\right] \mathbb{K}_{(1)}(\kappa)\right] \\
& -\frac{1}{\mathbb{E}\left[U_{(1)} \mid U_{(1)}<\kappa\right]} \int_{0}^{\kappa} \frac{v n[\overline{\mathbb{K}}(v)]^{n-1} g(v)}{1-[\overline{\mathbb{K}}(\kappa)]^{n}} \ln \left(v \varphi_{(1)}(v)\left[1-\overline{\mathbb{K}}^{n}(v)\right]\right) d v .
\end{aligned}
$$

$$
\begin{aligned}
\xi_{2}^{w}\left(U_{(1)}, \kappa\right)= & \ln \left(\mathbb{E}\left[U_{(1)} \mid U_{(1)}<\kappa\right]\left(1-\overline{\mathbb{K}}^{n}(\kappa)\right)\right) \\
& +\frac{\left.\int_{0}^{\overline{\mathbb{K}}(\kappa)} \overline{\mathbb{K}}^{-1}(u) n[u]^{n-1}\right) \ln \left(n \overline{\mathbb{K}}^{-1}(u) \frac{g\left(\overline{\mathbb{K}}^{-1}(u)\right)}{u\left((u)^{-n}-1\right)}\left(1-u^{n}\right)\right) d u}{\mathbb{E}\left[U_{(1)} \mid U_{(1)}>\kappa\right]\left(1-\overline{\mathbb{K}}^{n}(\kappa)\right)} .
\end{aligned}
$$

According to Eqs. (4-5) in Tavangar and Asadi [13], the mean PL of series system can be obtained as follows:

$$
\begin{gathered}
P_{1}(\kappa)=\mathbb{E}\left[\kappa-U_{(1)} \mid U_{(1)}<\kappa\right], \\
=\frac{\sum_{l=1}^{n}\left(\begin{array}{c}
n \\
l
\end{array}\right) \alpha_{\kappa}^{l} S_{l}(\kappa)}{\sum_{l=1}^{n}\left(\begin{array}{l}
n \\
l
\end{array}\right) \alpha_{\kappa}^{l}},
\end{gathered}
$$

where $\alpha()=.\mathbb{K}(.) / \overline{\mathbb{K}}($.$) and$

$$
S_{\pi}(\kappa)=\int_{0}^{\kappa} \sum_{l=1}^{\pi}\left(\begin{array}{l}
\pi \\
l
\end{array}\right)\left(\frac{\mathbb{K}(\kappa-v)}{\mathbb{K}(\kappa)}\right)^{l}\left(1-\frac{\mathbb{K}(\kappa-v)}{\mathbb{K}(\kappa)}\right)^{\pi-l} d v, 3.7
$$

Equations (17) and (18) demonstrate that

$$
\begin{aligned}
\xi_{2}^{w}\left(U_{(1)}, \kappa\right)= & \ln \left(\left(\kappa-P_{1}(\kappa)\right)\left(1-\overline{\mathbb{K}}^{n}(\kappa)\right)\right) \\
& +\frac{\left.\int_{0}^{\overline{\mathbb{K}}(\kappa)} \overline{\mathbb{K}}^{-1}(u) n[u]^{n-1}\right) \ln \left(n \overline{\mathbb{K}}^{-1}(u) \frac{g\left(\overline{\mathbb{K}}^{-1}(u)\right)}{u\left((u)^{-n}-1\right)}\left(1-u^{n}\right)\right) d u}{\left(\kappa-P_{1}(\kappa)\right)\left(1-\overline{\mathbb{K}}^{n}(\kappa)\right)} .
\end{aligned}
$$




\subsection{A Parallel Structure}

It is to be noted that $U_{(n)}$ refers to the lifetime of $P S$ with survival function $\overline{\mathbb{K}}_{(n)}()=.1-\mathbb{K}^{n}($.$) . Based on Eq. (13), we can define the weighted$ the residual entropy of $U_{(n)}$ as

$$
\begin{aligned}
\xi_{1}^{w}\left(U_{(n)}, \kappa\right)= & \ln \left[\mathbb{E}\left[U_{(n)} \mid U_{(n)}>\kappa\right]\left[1-\mathbb{K}^{n}(\kappa)\right]\right] \\
& -\frac{1}{\mathbb{E}\left[U_{(n)} \mid U_{(n)}>\kappa\right]\left[1-\mathbb{K}^{n}(\kappa)\right]} \int_{\kappa}^{\infty} v n \mathbb{K}^{n-1}(v) g(v) \ln \left(v n \mathbb{K}^{n-1}(v) g(v)\right) d v .
\end{aligned}
$$

Applying Theorem 2.1, pp. 477 in Asadi and Bayramoglu [14] and Eq. (18), it obtains that,

$$
\mathbb{E}\left[U_{(n)} \mid U_{(n)}>\kappa\right]=\mathbb{B}_{(n)}(\kappa)+\kappa,
$$

where $\mathbb{B}_{(n)}(\kappa)$ is mean residual lifetime of $P S$, it can be found as follows

$$
\mathbb{B}_{(n)}(\kappa)=\frac{\sum_{l=1}^{n-1}\left(\begin{array}{l}
n \\
l
\end{array}\right) \alpha^{l}(\kappa) \sum_{s=1}^{n}(-1)^{s-1}\left(\begin{array}{l}
n-l \\
s
\end{array}\right) \beta_{s}(\kappa)}{\sum_{l=1}^{n-1}\left(\begin{array}{l}
n \\
l
\end{array}\right) \alpha^{l}(\kappa)}
$$

and $\beta_{j}(\kappa)=\int_{\kappa}^{\infty} \frac{\overline{\mathbb{K}}^{j}(v) d v}{\overline{\mathbb{K}}^{j}(\kappa)}$. Now, it is evident that

$$
\begin{aligned}
\xi_{1}^{w}\left(U_{(n)}, \kappa\right)= & \ln \left[\left(\mathbb{B}_{(n)}(\kappa)+\kappa\right)\left[1-\mathbb{K}^{n}(\kappa)\right]\right] \\
& -\frac{n}{\left(\mathbb{B}_{(n)}(\kappa)+\kappa\right)\left[1-\mathbb{K}^{n}(\kappa)\right]} \int_{\kappa}^{\infty} v \mathbb{K}^{n-1}(v) g(v) \ln \left(v n \mathbb{K}^{n-1}(v) g(v)\right) d v
\end{aligned}
$$

By using Eq. (4) in Asadi (2006, pp. 1200), we have the mean PL of the components of PS as follows

$$
\theta_{n}(\kappa)=\mathbb{E}\left[\kappa-U_{(n)} \mid U_{(n)} \leq \kappa\right]=\int_{0}^{\kappa} \frac{\mathbb{K}^{n}(v) d v}{\mathbb{K}^{n}(\kappa)}
$$

Putting $r=n$ in Eq. (14) and using Eqs. (12 and 14), we get the WPE of $U_{(n)}$ as follows

$$
\begin{aligned}
\xi_{2}^{w}\left(U_{(n)}, \kappa\right) & =\ln \left[\left(\kappa-\theta_{n}(\kappa)\right) \mathbb{K}_{(n)}(\kappa)\right]-\frac{1}{\kappa-\theta_{n}(\kappa)} \int_{0}^{\kappa} \frac{v g_{(n)}(v)}{\mathbb{K}_{(n)}^{w}(\kappa)} \ln \left(v \varphi_{(n)}(v) \mathbb{K}_{(n)}(v)\right) d v \\
& =\ln \left[\left(\kappa-\theta_{n}(\kappa)\right) \mathbb{K}_{(n)}(\kappa)\right]-\frac{1}{\kappa-\theta_{n}(\kappa)} \int_{0}^{\kappa} \frac{v g_{(n)}(v)}{\mathbb{K}_{(n)}(\kappa) \mathbb{E}\left[U_{(n)} \mid U_{(n)}<\kappa\right]} \ln \left(v n \varphi_{\mathbb{K}}(v) \mathbb{K}^{n}(v)\right) d v,
\end{aligned}
$$

which is equivalent to,

$$
\begin{aligned}
\xi_{2}^{w}\left(U_{(n)}, \kappa\right)= & \ln \left[\left(\kappa-\theta_{n}(\kappa)\right) \mathbb{K}_{(n)}(\kappa)\right] \\
& -\frac{n}{\left(\kappa-\theta_{n}(\kappa)\right)\left(\mathbb{K}^{n}(\kappa)\left(\kappa-\theta_{n}(\kappa)\right)\right)} \int_{0}^{\kappa} v \mathbb{K}^{n-1}(v) g(v) \ln \left(v n \varphi_{\mathbb{K}}(v) \mathbb{K}^{n}(v)\right) d v,
\end{aligned}
$$

for all $\kappa \geq 0$. 


\subsection{Some inequalities}

Next, we derive the upper bound of WRE of $U_{(r)}$. It is obvious that

$$
\begin{aligned}
\xi_{1}^{w}\left(U_{(r)}, \kappa\right)= & \ln \mathbb{E}\left[U_{(r)} \mid U_{(r)}>\kappa\right]+\ln \overline{\mathbb{K}}_{(r)}(\kappa) \\
& -\frac{1}{\mathbb{E}\left[U_{(r)} \mid U_{(r)}>\kappa\right]} \int_{\kappa}^{\infty} \frac{v g_{(r)}(v)}{\overline{\mathbb{K}}_{(r)}(\kappa)} \ln \left(v \bar{\varphi}_{(r)}(v) \overline{\mathbb{K}}_{(r)}(v)\right) d v,
\end{aligned}
$$

since $\ln \mathbb{E}\left[U_{(r)} \mid U_{(r)}>\kappa\right] \geq 0$ and

$$
\kappa \geq 0 \Rightarrow \ln \left[\overline{\mathbb{K}}_{(r)}(\kappa)\right] \leq 0,
$$

by using Gupta et al. [16], we can deduce that

$$
\xi_{1}^{w}\left(U_{(r)}, \kappa\right) \leq \ln \mathbb{E}\left[U_{(r)} \mid U_{(r)}>\kappa\right]
$$

For $r=1$, we have

$$
\xi_{1}^{w}\left(U_{(1)}, \kappa\right) \leq \ln \mathbb{M}_{1}(\kappa)
$$

In addition, we know that $\ln \left[\left(\mathbb{B}_{(n)}(\kappa)+\kappa\right)\left[1-\mathbb{K}^{n}(\kappa)\right]\right] \leq 0$. Hence, we have

$$
\xi_{1}^{w}\left(U_{(n)}, \kappa\right) \leq \ln \left[\left(\mathbb{B}_{(n)}(\kappa)+\kappa\right)\left[1-\mathbb{K}^{n}(\kappa)\right]\right] .
$$

In next result, we derive the lower bound for WPE of $U_{(n)}$.

Proposition 3.1: Suppose $U \geq 0$ be a random variable with distribution function $\mathbb{K}(v)$. Then

$$
\xi_{2}^{w}\left(U_{(n)}, \kappa\right) \geq \frac{-n^{2} E\left[u^{2} \mathbb{K}^{2 n-2}(u) g(u) \mid U \leq \kappa\right]}{\left(\kappa-\theta_{n}(\kappa)\right)^{2} \mathbb{K}^{n-1}(\kappa)} .
$$

Proof. Using Eq.(2), inequality $-\ln y \geq 1-y$, for $y \geq 0$, and since

$$
\int_{0}^{\kappa} v \mathbb{K}^{n-1}(v) g(v) d v \leq \int_{0}^{\kappa} n v^{2} \mathbb{K}^{2 n-2}(v) g^{2}(v) d v .
$$

The result follows.

\section{STOCHASTIC ORDERS BASED ON WEIGHTED ENTROPY}

In this section, we explore the possibility of application of stochastic orders.

Definition 4.1. Assume $U_{1} \geq 0$ and $U_{2} \geq 0$ be two random variables with density functions $g_{1}$ and $g_{2}$, distribution functions $\mathbb{G}_{U_{1}}$ and $\mathbb{G}_{U_{2}}$, reliability functions $\overline{\mathbb{G}}_{U_{1}}=1-\mathbb{G}_{U_{1}}$ and $\overline{\mathbb{G}}_{U_{2}}=1-\mathbb{G}_{U_{2}}$, the weighted entropy functions $\xi_{g_{1}}^{w}($.$) and \xi_{g_{2}}^{w}($.$) , the convex residual entropy functions$ $H_{g_{1}}^{w}\left(U_{1}, t\right)$ and $H_{g_{2}}^{w}\left(U_{2}, t\right)$ and the weighted inaccuracy measures $\mathbb{R}\left(U_{1}^{w} \| U_{1}\right)$ and $\mathbb{R}\left(U_{2}^{w} \| U_{2}\right)$, respectively. We say that $U_{1}$ is $\mathbb{S} \mathbb{E}$ to $U_{2}$ in the:

- weighted entropy ordering $\left(U_{1} \leq_{\xi^{w}} U_{2}\right)$ if $\xi_{g_{1}}^{w}(x) \leq \xi_{g_{2}}^{w}(x)$, for all $x \geq 0$.

- weighted inaccuracy ordering $\left(U_{1} \leq_{\mathbb{R}^{w}} U_{2}\right)$ if $\mathbb{R}\left(U_{1}^{w} \| U_{1}\right) \leq \mathbb{R}\left(U_{2}^{w} \| U_{2}\right)$.

- convex residual entropy ordering $\left(U_{1} \leq_{c^{w}} U_{2}\right)$ if $H_{g_{1}}^{w}\left(U_{1}, t\right) \leq H_{g_{2}}^{w}\left(U_{2}, t\right)$.

- less uncertainty ordering $\left(U_{1} \leq_{\mathbb{U}} U_{2}\right)$ if $H\left(U_{1}\right) \leq H\left(U_{2}\right)$.

Definition 4.2. Let $U_{1}$ and $U_{2}$ be two random variables, then $U_{1}$ is said to be $\mathbb{S E}$ to $U_{2}$ in the convex order $\left(U_{1} \leq_{c x} U_{2}\right)$. If

$$
E\left[\phi\left(U_{1}\right)\right] \leq E\left[\phi\left(U_{2}\right)\right],
$$

This is true for any convex functions $\phi$. 
Definition 4.3. The random variable $U_{1}$ is said to be increasing hazard rate, $I H R$, if, and only if,

$$
\overline{\mathbb{G}}_{U_{1}}(u+v) / \overline{\mathbb{G}}_{U_{1}}(u) \text { is decreasing in } u \geq 0 \text {, for all } v \geq 0 .
$$

Next result discusses the closure under increasing linear transformation of $\leq_{\xi^{w}}$ :

Theorem 4.1. Suppose $U_{1}$ and $U_{2}$ are to be two random variables, let we define new functions as

$$
V_{1}=\alpha_{1} U_{1}^{\beta_{1}} \text { and } V_{2}=\alpha_{2} U_{2}^{\beta_{2}} \text {, for all } \alpha_{1}, \alpha_{2} \in \mathbb{R}^{+} \text {and } \beta_{1}, \beta_{2} \in \mathbb{R}^{+} .
$$

Let (i) $U_{1} \leq_{\xi^{w}} U_{2}$, (ii) $\alpha_{1} \leq \alpha_{2}$, (iii) $\beta_{1} \leq \beta_{2}$. Then $V_{1} \leq_{\xi^{w}} V_{2}$ if $U_{1} \leq_{c x} U_{2}$.

Proof. Due to fact that $\varphi(x)=x \ln x$ is convex function, and when $U_{1} \leq_{c x} U_{2}$ we get that $E\left[U_{1}\right]=E\left[U_{2}\right]$, if we suppose that $\xi_{U_{1}}^{w}(u)$ is decreasing in $u$, and let $U_{1} \leq_{\xi^{w}} U_{2}, \alpha_{1} \leq \alpha_{2}$ and $\beta_{1} \leq \beta_{2}$. By apply Eq. (7) and Proposition 2.3, we have $V_{1} \leq \xi^{w} V_{2}$.

Corollary 4.1. Suppose the relationship between two random variables $U_{1}$ and $U_{2}$ as follows:

$$
U_{1} \leq_{\xi^{w}} U_{2}
$$

Define $V_{1}=\alpha U_{1}^{\beta}$ and $V_{2}=\alpha U_{2}^{\beta}, \alpha, \beta \in \mathbb{R}^{+}$. Then $U_{1} \leq_{\xi^{w}} U_{2}$ if $U_{1} \leq_{c x} U_{2}$.

In next theorem, we explain preservation properties and application of $\leq_{\xi^{w}}, \leq_{\mathbb{R}^{w}}$ and $\leq_{\mathbb{U}}$ between two exponential RV's if their scale parameters are ordered.

Theorem 4.2. Let two absolutely continuous random variables $U_{1}$ and $U_{2}$ with density function

$$
f_{i}(x)=\alpha_{i} \exp \left(-\alpha_{i} x\right), \alpha_{i}, x>0 \text { and } i=1,2 .
$$

- If $\alpha_{1} \geq \alpha_{2}$, then $U_{1} \leq_{\xi^{w}} U_{2}$.

- If $\alpha_{1} \leq \alpha_{2}$, then $U_{1} \leq_{\mathbb{R}^{w}} U_{2}$.

- If $X \leq_{\mathbb{R}^{w}} Y$ then $H_{U_{2}} \leq_{\mathbb{U}} H_{U_{1}}$.

Proof. The result is obtained immediately from Remark 2.1 .

Many studies explain the properties of repairable systems such as minimal repair. If the system has the virtual age $\mathbb{U}_{n-1}$ immediately after the $(n-1)^{\text {th }}$ repair, the functioning system obtained has the $n$th failure-time $\mathbb{Y}_{n}$ distributed as

$$
\operatorname{Pr}\left[\mathbb{Y}_{n} \leq y \mid \mathbb{U}_{n-1}=u\right]=\frac{G(y+u)-G(u)}{\bar{G}(u)},
$$

where $G(y)$ is the failure time distribution of a new system $\left(\mathbb{U}_{0}=0\right)$. Let $n^{\text {th }}$ repair cannot remove the damages incurred before the $(n-1)^{\text {th }}$ repair and $\alpha_{n}$ be the degree of the $n^{\text {th }}$ repair, now the time between $(n-1)^{\text {th }}$ failure and nth failure reduce from $\mathbb{Y}_{n}$ to $\alpha_{n} \mathbb{Y}_{n}$. If $\alpha_{n}=1$ for all $n \geq 1$ then it agrees with a minimal repair model.

Suppose $\mathbb{V}_{n}=\sum_{i=1}^{n} \mathbb{Y}_{i}(n \geqq 1)$ with $\mathbb{V}_{0}=0$ which represents the time elapsed since the system was put in operation, or the associated counting process $\mathbb{N}(t)=\sup \left\{n \geqq 1: \mathbb{V}_{n-1} \leqq t\right\}$. Kijima [17] proved that $\mathbb{N}(t)\left(\right.$ or $\left.\left\{\mathbb{V}_{n}\right\}_{0}^{\infty}\right)$ is a non-homogeneous Poisson process when $\alpha_{n}=1$ for all $n \geq 1$. Ebrahimi and Pellerey [1] defined the following definition:

Definition 4.4. A point process $\{\mathbb{N}(t), t \geq 0\}$ consisting of interarrival times $\mathbb{Y}_{1}, \mathbb{Y}_{2}, \ldots$ is increasing (decreasing) in the

1. convex residual entropy order if

$$
H^{w}\left(\mathbb{B}_{i}, t\right) \leq(\geq) H^{w}\left(\mathbb{B}_{j}, t\right), \text { for all } t \in R^{+}
$$

2. weighted entropy order if

$$
\xi^{w}\left(\mathbb{B}_{i}, t\right) \leq(\geq) \xi^{w}\left(\mathbb{B}_{j}, t\right)
$$

and $1 \leq i \leq j \leq n$, where $f_{k}$ is the conditional probability density function of $\mathbb{V}_{k}=\mathbb{V}_{k}-\mathbb{V}_{k-1}$ for all $k=1,2, \ldots$, given $\mathbb{V}_{k-1}=v_{k-1}, \ldots, \mathbb{V}_{1}=v_{1}$, and

$$
\mathbb{B}_{k} \stackrel{s t}{=}\left[\mathbb{Y}_{k} \mathbb{\mathbb { V } _ { k - 1 }}=v_{k-1}, \ldots, \mathbb{V}_{1}=v_{1}\right]
$$


From Definition 5.3, we can note that if a point process is increasing (decreasing) means the uncertainty of the distribution is increasing (decreasing), i.e., the process is deterioration (improving).

Lemma 4.1. Let $\mathbb{B}_{k}$ be as defined in Definition 5.3. Then, for $k=1,2, \ldots$,

1. $H^{w}\left(\mathbb{B}_{k}, t\right)=H^{w}\left(X, t+v_{k-1}\right)$;

2. $\xi^{w}\left(\mathbb{B}_{k}, t\right)=\xi^{w}\left(X, t+v_{k-1}\right)$.

Proof. Refer to Ebrahimi and Pellery [1], Theorem 2.5.

Theorem 4.3. The stochastic point process $\mathbb{N}(t)=\sup \left\{n \geqq 1: \mathbb{V}_{n} \leqq t\right\}$ consisting of the time of $n t h$ failure $\mathbb{V}_{k-1}, k=1,2, \ldots$ generated by a minimal repair policy is increasing (decreasing) in

1. convex residual entropy order if $G($.$) is I H R$,

2. weighted entropy order if $\xi^{w}(U)$ is increasing for all $u \geq 0$.

Proof. Similarly to lemma 5.1, we have $H^{w}\left(\mathbb{B}_{k+1}, t\right)=H^{w}\left(U, t+v_{k}\right)$. In addition, by Theorem 3.1 in Di Crescenzo and Longobardi [4] we conclude that

$$
H^{w}\left(U, t+v_{k}\right)=\left(t+v_{k}\right)\left[1-\ln \lambda\left(t+v_{k}\right)\right]+\frac{t+v_{k}}{\lambda\left(t+v_{k}\right)} \frac{d}{d t} H\left(U, t+v_{k}\right)+\frac{1}{\bar{G}\left(t+v_{k}\right)} I\left(t+v_{k}\right), 4.2
$$

where

$$
I(t)=\int_{t}^{\infty} \bar{G}(u) H(U, u) d u-\int_{t}^{\infty} \bar{G}(u) \ln \frac{\bar{G}(u)}{\bar{G}(t)} d u
$$

and by Theorem 2.1 in Ebrahimi and Pellery [1] we have

$$
H^{w}\left(U, t+v_{k}\right)=\left(t+v_{k}\right) H\left(U, t+v_{k}\right)+\frac{1}{\bar{G}\left(t+v_{k}\right)} I\left(t+v_{k}\right) .4 .3
$$

By Theorem 2.5 in Ebrahimi and Pellery [1] we conclude that $H\left(U, t+v_{n-1}\right) \leq H\left(U, t+v_{n}\right)$. By using Eqs. (20-22), and when a continuous distribution $G($.) is IHR. It is obvious that

$$
\bar{G}\left(t+v_{k}\right) \leq \bar{G}\left(t+v_{k-1}\right),
$$

and

$$
I\left(t+v_{k}\right) \geq I\left(t+v_{k-1}\right)
$$

Then, we get $H^{w}\left(U, t+v_{k}\right) \geq H^{w}\left(U, t+v_{k-1}\right)$. This complete the proof.

\section{ENTROPY ESTIMATION}

In this section, we introduce four the non-parametric estimators of Eq. (6) by using the same idea in Vasicek [9], Van Es [18], Ebrahimi $e t$ al. [19] and Al-Omari [20].

Let $Z_{1}, Z_{2}, \ldots, Z_{n}$ is a sequence of the random sample with the distribution $G$ and let $Z_{(1)}, Z_{(2)}, \ldots, Z_{(n)}$ be the corresponding order statistics. Besides the sample distribution function $G_{n}(z)=n^{-1} \sum_{i=1}^{n} \mathbf{1}_{Z_{k} \leq z, 1 \leq k \leq n}$. Then, the on-parametric estimators can be expressed as:

1. Weighted Vasicek Entropy $\left(V \xi_{(\delta, n)}^{w}(Z)\right)$ : We can estimate of Eq. (8) by replacing $G^{w}(t)$ by the empirical distribution $G_{n}^{w}(t)$, and using a difference operator in place of the differential operator. Thus, $V \xi_{(m, n)}^{w}(Z)$ estimator of Eq. (8) can be represented as follows

$$
V \xi_{(\delta, n)}^{w}(Z)=n^{-1} \sum_{i=1}^{n} \ln \left\{\frac{n \sum_{k=1}^{n} w\left(Z_{(k)}\right)}{\sum_{j=1}^{i} w\left(Z_{(j)}\right) 2 \delta}\left(Z_{(i+\delta)}-Z_{(i-\delta)}\right)\right\},
$$


where $\delta \in \mathbb{N}^{+}$know as a window size, $\delta<n / 2, Z_{(s)}=Z_{(1)}$ if $s<1$ and $Z_{(s)}=Z_{(n)}$ if $s>n$.

2. Weighted $\delta$-spacings Entropy $\left(S E \xi_{(\delta, n)}^{w}(Z)\right)$ : Estimates of weighted entropy based on sample $\delta$-spacings which introduced by Van Es [18], we can provide $V E \xi_{(\delta, n)}^{w}(Z)$ estimator of Eq. (6) as

$$
S E \xi_{(\delta, n)}^{w}(Z)=n^{-1} \sum_{i=1}^{n-\delta} \ln \left\{\frac{n \sum_{j=1}^{i} Z_{(j)}}{\sum_{k=1}^{n} Z_{(k)} \delta}\left(Z_{(i+\delta)}-Z_{(i)}\right)\right\}-\psi(\delta)+\ln \delta+E[Z]
$$

where $\psi($.$) is the digamma function and \ln \delta-\psi(\delta)$ corrected bias entropy estimator.

3. Weighted Small weights Entropy $\left(W S \xi_{(\delta, n)}^{w}(Z)\right)$ : As assign smaller weights in Vasicek [9], we obtain

$$
W S \xi_{(\delta, n)}^{w}(Z)=n^{-1} \sum_{i=1}^{n} \ln \left\{\frac{n \sum_{k=1}^{n} Z_{(k)}}{\sum_{j=1}^{i} Z_{(j)} \alpha_{i} \delta}\left(Z_{(i+\delta)}-Z_{(i-\delta)}\right)\right\}
$$

where

$$
\alpha_{k}= \begin{cases}\frac{\delta+k-1}{\delta}, & 1 \leq k \leq \delta \\ 2, & \delta+1 \leq k \leq n-\delta \\ \frac{\delta+n-k}{\delta}, & n-\delta+1 \leq k \leq n\end{cases}
$$

4. Modified Small weights Entropy $\left(M S \xi_{(\delta, n)}^{w}(Z)\right)$ : As assign smaller weights in Ibrahimi et al. [21] we get

$$
M S \xi_{(\delta, n)}^{w}(Z)=n^{-1} \sum_{i=1}^{n} \ln \left\{\frac{n \sum_{k=1}^{n} w\left(Z_{(k)}\right)}{\sum_{j=1}^{i} w\left(Z_{(j)}\right) \beta_{i} \delta}\left(Z_{(i+\delta)}-Z_{(i-\delta)}\right)\right\}
$$

where

$$
\beta_{i}= \begin{cases}\frac{3}{2}, & 1 \leq i \leq \delta \\ 2, & \delta+1 \leq i \leq n-\delta \\ \frac{1}{2}, & n-\delta+1 \leq i \leq n\end{cases}
$$

Example 5.1: Let $Z$ be random variable having exponential distribution with density function $f_{Z}(x)=\alpha \exp (-\alpha z), \alpha, z>0$ with parameter $\alpha>0$. From Eq. (7) we obtain,

$$
\vartheta^{w}(Z)=\frac{1}{\alpha}(\psi(2)-\ln \alpha)
$$

where $\psi($.$) is Euler's psi function. By Example (2.1, a)$ in Di Crescenzo and Longobardi [4] we get,

$$
H^{w}(Z)=\frac{2-\ln \alpha}{\alpha} \text { and } \xi_{1}^{w}(Z)=2-\ln \alpha-\psi(2) .
$$


Therefore,

$$
\mathbb{R}\left(Z^{w} \| Z\right)=-4(1-\ln \alpha)-\psi(2)=-4 H_{Z}-\psi(2) .
$$

Suppose $\alpha \in[0,100] . H^{w}(Z), \xi^{w}(Z)$ and $\mathbb{R}\left(Z^{w} \| Z\right)$ with weighted function $w(z)=z$ is evaluated in Table 1 .

Table 1 Measures of weighted entropies of exponential distribution.

\begin{tabular}{llll}
\hline$\alpha$ & $\hat{H}^{w}(Z)$ & $\hat{\xi}^{w}(Z)$ & $\hat{\mathbb{R}}^{w}\left(Z^{w} \| Z\right)$ \\
\hline 0.25 & 13.5452 & 2.9635 & -9.9680 \\
0.5 & 5.3863 & 2.2704 & -7.1954 \\
1 & 2 & 1.5772 & -4.4228 \\
5 & 0.0781 & -0.0322 & 2.0150 \\
10 & -0.0303 & -0.7254 & 4.7876 \\
\hline
\end{tabular}

Now, a real data is illustrated to investigate the performance of suggested estimators.

Data Set: The following data set which is taken from Smith and Naylor [22], it represents the strength of $1.5 \mathrm{~cm}$ glass fibers measured at the National Physical Laboratory, England.

Data Set: $0.55,0.93,1.25,1.36,1.49,1.52,1.58,1.61,1.64,1.68,1.73,1.81,2.00,0.74,1.04,1.27,1.39,1.49,1.53,1.59,1.61,1.66,1.68,1.76$, $1.82,2.01,0.77,1.11,1.28,1.42,1.50,1.54,1.60,1.62,1.66,1.69,1.76,1.84,2.24,0.81,1.13,1.29,1.48,1.50,1.55,1.61,1.62,1.66,1.70,1.77$, $1.84,0.84,1.24,1.30,1.48,1.51,1.55,1.61,1.63,1.67,1.70,1.78,1.89$

Shanker et al. [23] show the exponential density function $(\operatorname{Exp}(0.663647))$ provided a better fit for this data. We compute the exact value of the weight entropy measure by the real data and compare this measure with $V \xi_{(\delta, n)}^{w}(Z), S E \xi_{(\delta, n)}^{w}(Z), W S \xi_{(\delta, n)}^{w}(Z)$ and $M S \xi_{(\delta, n)}^{w}(Z)$ which shows in Table 2.

Table 2 Weighted entropy measure for exponential distribution (0.663647).

\begin{tabular}{cccccc}
\hline$\delta$ & $\xi_{(\delta, n)}^{w}(Z)$ & $V \xi_{(\delta, n)}^{w}(Z)$ & $S E \xi_{(\delta, n)}^{w}(Z)$ & $W S \xi_{(\delta, n)}^{w}(Z)$ & $M S \xi_{(\delta, n)}^{w}(Z)$ \\
\hline 1 & 1.9872 & 1.210967749 & 1.244680937583 & 1.232972422 & 1.237538804 \\
2 & 1.9872 & 1.200196229 & 1.231653171583 & 1.943017269 & 1.298445816 \\
3 & 1.9872 & 1.245455104 & 1.462350850583 & 2.308545839 & 1.323498873 \\
4 & 1.9872 & 1.279410563 & 1.650373401583 & 2.537544185 & 1.337842382 \\
5 & 1.9872 & 1.282220574 & 1.820823663583 & 2.721711858 & 1.415075846 \\
\hline
\end{tabular}

\section{REFERENCES}

1. N.Ebrahimi, and F. Pellerey. J. App. Prob. 32. 1. 1995. 202-211.

2. G.Asha, C.J.Rejeesh, Metron. 73. 1. 2015. 119-134.

3. Khinchin, A.I. (1957). Mathematical Foundation of Information Theory. Dover Publications, New York.

4. Di Crescenzo, M. Longobardi, Sci. Math. Japn. 64(2) (2006), 679-690.

5. Di Crescenzo, M. Longobardi,J. Appl. Probab.. 39. 2. (2002). 434-440.

6. S. Kullback, R.A. Leibler,Ann. Math. Stat.22(1) (1951),79-86.

7. N. Ebrahimi, S.N.U.A. Kirmani,Ann. Inst. Stat. Math.48(2) (1996),257-265.

8. S. Das,Commun. Stat. Theory Methods.46(12) (2017),5707-5727.

9. O.Vasicek, J. R. Stat. Soc. Ser. B. 38. (1). (1976). 54-59.

10. I.S. Gradshteyn, I.M.Ryzhik, Table of Integrals, Series and Products, fifth ed. Academic Press New York 1994.

11. I.Bairamov, M.Ahsanullah, I.Akhundov, J. Stat. Theory Appl. 1. 2. (2002). 119-132.

12. M.Asadi, I.Bayramoglu, IEEE. Trans. Reliab.. 55. 2. (2006). 314-318.

13. M.Tavangar, M.Asadi, Metrika. 72. 1. (2010). 59-73.

14. M. Asadi, I. Bayramoglu,Commun. Stat. Theory Methods.34(2) (2005),475-485.

15. M.Asadi. J. Stat. Plan. Infer. 136. 4. (2006). 1197-1206.

16. R.C.Gupta, H.C.Taneja, R.Thapliyal, Stat. Theory Appl. 13. (1). (2014). 27-37.

17. M.Kijima, J. Appl. Probab. 26. (1). (1989). 89-102.

18. B.Van Es. Scand. J. Stat. 19(1) (1992). 61-72.

19. N. Ebrahimi, K. Pflughoeft, E. Soofi,Stat. Probab. Lett.20(3) (1994),225-234.

20. A.I.Al-Omari, J. Comput. Appl. Math.. 261. (1). (2014). 95-102.

21. Ibrahimi et al. (1994).

22. R.L.Smith, J.C.Naylor. J. R. Stat. Soc.. 36. (3). (1987). 358-369.

23. R. Shanker, H. Fesshaye, S. Selvaraj,Biom. Biostat. Int. J.2(5) (2015),1-9. 